FACULDADE DE CIÊNCIAS ECONÔMICAS DA UFRGS Análise
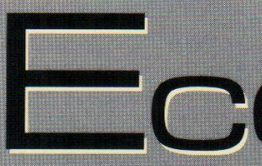

VALORES E PREÇOS DE PRODUÇÃO: UMA RELETURA de MARX Alfredo SAAD FILHO

OS ANTIGOS, OS NOVOS E OS NEO-INSTITUCIONALISTAS: HÁ CONVERGÉ NCIA TEÓ RICA NO PENSAMENTO INSTITUCIONALISTA

Octavio Augusto C. Conceição

A MENSURAÇÃ O DO GRAU DE INDEPENDÉNCIA DO BANCO CENTRAL: UMA ANÁLISE DE SUAS FRAGILIDADES HeLder Ferreira de MENDONÇA

A SUSTENTABILIDADE DA DÍVIDA MOBILIÁ RIA FEDERAL BRASILEIRA: UMA INVESTIGAÇĀ O ADICIONAL VIVIANE LUPORINI

O MODELO dE KRUGMAN EXPLICA A CRISE CAMBIAL. BRASILEIRA EM JANEIRO DE 1999?

Adriano CAMPOS MENEZES E TITO BELCHIOR S. MOREIR/

ESTRANGULAMENTO DA díVIDA EXTERNA E CRESCIMENTO ECONO MICO NA AMÉRICA LATINA: LIÇÓ ES DA DÉCADA DE 80

Marco Vaies buratto E SABino da SiLva P. JR.

CriaÇÃo e desvio de COMÉrCio: ANÁlise do FLuXo COMERCial ENTRE O Brasil. e O MERCOSUl para Alguns PRODUTOS INDUSTRIALIZADOS

Clá Udio Roberto fó fFano Vasconcelos

A EVOLUCÁ̃ DA INDÚ STRIA BRASILEIRA DE CELULOSE E SUA ATUAÇÃO NO MERCADO MUNDIAL

Emerson Martins HILGemberg e Carlos josé Caetano BACHA

A PROTEÇÃo SOCIAL NO PRIMEIRO QUARTEL DO SÉCULO XXI: BRINCANDO COM CENÁRIOS

rosa Maria Marques e Áquilas Mendes

ECONOMIA DO CRIME: ELEMENTOS TEÓ RICOS E EVIDÊNCIAS EMPÍRICAS

Gilberto José SChaefer e Pery Francisco Assis Shikida

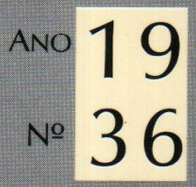


UNIVERSIDADE FEDERAL DO RIO GRANDE DO SUL

Reitora: Profa. Wrana Maria Panizzi

FACULDADE DE CIÊNCIAS ECONÔMICAS

Diretor: Prof. Pedro Cézar Dutra Fonseca

CENTRO DE ESTUDOS E PESQUISAS ECONÔMICAS

Diretor: Prof. Gentil Corazza

DEPARTAMENTO DE CIÊNCIAS ECONÔMICAS

Chefe: Prof. Luiz Alberto Oliveira Ribeiro de Miranda

DAPARTAMENTO DE CIÊNCIAS CONTÁBEIS E ATUARIAIS

Chefe: João Marcos Leão da Rocha

CURSO DE PÓS-GRADUAÇÃO EM ECONOMIA

Coordenador: Prof. Eduardo Pontual Ribeiro

CURSO DE PÓS-GRADUAÇÃO EM ECONOMIA RURAL

Coordenador: Prof. Jalcione Almeida

CONSELHO EDITORIAL: Achyles B. Costa, Aray M. Feldens, Carlos A. Crusius, Carlos G. A. Mielitz Netto, Eduardo A. Maldonado Filho, Eduardo P. Ribeiro, Eugênio Lagemann, Fernando Ferrari Filho, Gentil Corazza, Jan A. Kregel (Univ. of Bologna), Marcelo S. Portugal, Nali J. Souza, Otilia B. K. Carrion, Paulo A. Spohr, Paulo D. Waquil, Pedro C. D. Fonseca, Philip Arestis (Univ of East London), Roberto C. Moraes, Ronald Otto Hillbrecht, Stefano Florissi, Eleutério F. S. Prado (USP), Fernando H. Barbosa (FGV/RJ), Gustavo Franco (PUC/RJ), João R. Sanson (UFSC), Joaquim P. Andrade (UnB), Juan H. Moldau (USP), Paul Davidson (Univ. of Tennessee), Werner Baer (Univ. of Illinois).

COMISSÃO EDITORIAL: Eduardo Augusto Maldonado Filho, Fermando Ferrari Filho, Gentil Corazza, Marcelo Savino Portugal, Paulo Dabdab Waquil; Roberto Camps Moraes.

EDITOR: Fernando Ferrari Filho

EDITOR ADJUNTO: Pedro Silveira Bandeira

SECRETÁRIA: Vanessa Hoffmann de Quadros

REVISÃO DE TEXTOS: Vanete Ricacheski

FUNDADOR: Prof. Antônjo Carlos Santos Rosa

Os materiais publicados na revista Análise Econômica sāo da exclusiva responsabilidade dos autores. É permitida a reproduçāo total ou parcial dos trabalhos, desde que seja citada a fonte. Aceita-se permuta com revistas congêneres. Aceitam-se, também, livros para divulgação, elaboração de resenhas e recensões. Toda correspondência, material para publicação (vide normas na terceira capa), assinaturas e permutas devem ser dirigidos ao seguinte destinatário:

PROF. FERNANDO FERRARI FILHO

Revista Análise Econômica - Av. João Pessoa, 52 CEP 90040-000 PORTO ALEGRE - RS, BRASIL Telefones: (051) 316-3348 e 316-3440 - Fax: (051) 316-3990

E-mail: rae@vortex.ufrgs.br

Análise Econômica

Ano 18, n⿳3 33, março, 2000 - Porto Alegre

Faculdade de Ciências Econômicas, UFRGS, 2000

Periodicidade semestral, março e setembro.

1. Teoria Econômica - Desenvolvimento Regional -

Economia Agrícola - Pesquisa Teórica e Aplicada -

Periódicos. I. Brasil

Faculdade de Ciências Econômicas,

Universidade Federal do Rio Grande do Sul.

CDD 330.05

CDU 33 (81) (05) 


\title{
A mensuração do grau de independência do Banco Central: uma análise de suas fragilidades
}

Helder Ferreira de Mendonça *

\begin{abstract}
Resumo: A discussão que envolve a independência dos bancos centrais apresenta dois pilares de sustentação: um teórico e outro empírico. $\mathrm{O}$ argumento teórico baseia-se na hipótese de que policymakers são sujeitos ao viés inflacionário. No que se refere ao caráter empírico, o ponto principal da discussão resulta da observação da existência de correlação negativa entre o grau de independência do banco central e a inflação. A análise empírica recebeu grande impulso a partir da segunda metade da década de 80 e início dos anos 90 com o desenvolvimento de índices de independência. Este artigo apresenta as fragilidades associadas aos índices que medem o nivel de independencia dos bancos centrais, e ilustra o caso do Brasil. Os resultados encontrados indicam que maior independência, tal como indicado pelos índices existentes, não é capaz de garantir baixas taxas de inflação.
\end{abstract}

Palavras-chave: independência dos bancos centrais; indices de independência; inflação; viés inflacionário.

\begin{abstract}
The discussion about the independence of central banks is based on theoretical arguments and empirical evidence. The theoretical argument has been based on the hypothesis that policymakers are subject to the inflation bias. The main empirical argument is that there is a negative correlation between the degree of independence of central banks and inflation. The empirical analysis has gained more interest since the second half of the $80 \mathrm{~s}$ and the beginning of the $90 \mathrm{~s}$ with the development of independence indexes. This article demonstrates the fragility of the indexes that measure the level of independence of central banks and illustrates the case of Brazil. The findings denote that an increase of independence, as indicated by the indexes, is not capable of guaranteeing low rates of inflation.
\end{abstract}

Key words: independence of central banks; indexes of independence; inflation; inflation bias.

JEL Classification: E52, E58.

- Professor do Departamento de Economia da UFF. 


\section{Introdução}

O significado do termo independência tem aparecido na maior parte da literatura, como a capacidade do banco central (BC) em não ceder frente às forças políticas para monetizar grandes déficits orçamentários. Ou seja, há a necessidade de independência institucional em relação aos poderes Executivo e Legislativo. Sendo assim, a presença de um banco central independente (BCI) em uma economia, teria, a priori, uma dupla vantagem: acabaria com as pressões advindas da esfera política (fonte do viés inflacionário), e faria com que o $\mathrm{BC}$ pudesse se concentrar em sua meta natural - a busca da estabilidade de preços.

$\mathrm{Na}$ década de 90, diversos países têm concedido maior autonomia aos seus bancos centrais devido às evidências apresentadas por vários economistas de que maior independência seria capaz de promover a estabilidade de preços ${ }^{1}$.

Economists and practitioners in the area of monetary policy generally believe that the degree of independence of the central bank from other parts of government affects the rates of expansion of money and credit and, through them, important macroeconomic variables, such as inflation and the size of the budget deficit. (Cukierman, Webb e Neyapti, 1992, p. 353-354).

A proposição de independência dos bancos centrais apresenta dois pilares de sustentação, um teórico e outro empírico. O argumento teórico apóia-se na visão de que policy-makers são sujeitos ao viés inflacionário ${ }^{2}$ intrínseco à condução da política monetária. A existência do viés inflacionário é resultado dos defensores da independência do $\mathrm{BC}$ admitirem a validade da taxa natural de desemprego ${ }^{3}$ e a neutralidade da moeda, ou

\footnotetext{
${ }^{1}$ Exemplos de países que caminham nessa direção são fartos, dentre os diversos casos destacamse: Nova Zelândia, Chile, México, Argentina, Espanha, França, Reino Unido, Canadá, Suécia, Austrália, Finlândia e Israel.

${ }^{2} \mathrm{O}$ conceito viés inflacionário deriva do argumento de ineficácia das políticas. $\mathrm{O}$ âmago do concejto pode ser entendido como a tentação que os governos possuem de buscar um aumento do produto e/ou redução do nível de desemprego por meio do uso de políticas monetárias expansionistas. Em outras palavras, os governos são propensos a fazer uso do chamado princípio aceleracionista da curva de Phillips (versão Friedman-Phelps), cujo resultado de longo prazo seria apenas um aumento da taxa de inflaçāo. Daí o termo viés inflacionário - má condução da política monetária que acarreta como único resultado maior inflaçāo.

${ }^{3}$ A taxa natural de desemprego " [....] is the level that would be ground out by the Walrasian system of general equilibrium equations, provided there is imbedded in them the actual structural characteristics of the labor and commodity markets, including market imperfections, stochastic variability in demands and supplies, the cost of gathering information about job vacancies and labor availabilities, the costs of mobility, and so on." (Friedman, 1968, p. 8).
} 
seja, não é adequado a tentativa do uso da política monetária como instrumento capaz de afetar variáveis reais da economia (alto nivel de emprego, financiamento do déficit orçamentário, baixas taxas de juros, etc.).

Para que se possa compreender o porquê do viés inflacionário, é importante que se faça uma distinção entre a perspectiva política e a econômica. Sob o ponto de vista político, os governos em períodos próximos às eleições tendem a preferir maior nível de emprego à menor taxa de inflação, pois acreditam que dessa forma conseguirão se reeleger ou favorecer a eleição dos candidatos de seu partido político. Nesse sentido, há uma tendência de os governos pressionarem os policy-makers para que implementem políticas que visam ao aumento do produto da economia. Isto implica dizer que, se houver na economia um BC subserviente, existe uma propensão a expandir a oferta monetária com o objetivo de reduzir o nível de desemprego.

Sob a óptica econômica, a análise pode ser compreendida por meio da estrutura da curva de Phillips. Na busca de um menor nível de desemprego (uma taxa de desemprego abaixo da natural), a autoridade monetária aumenta a oferta de moeda de forma inesperada, causando erro de expectativa do público quanto ao nível de preços. Devido a esse erro de estimativa, os trabalhadores decidem ofertar maior quantidade de mãode-obra ${ }^{4}$. Não obstante a redução do nível de desemprego, a própria dinâmica da economia leva os trabalhadores a refazerem suas expectativas tendo em conta o erro de previsão do período anterior. O resultado final é que a expansão monetária não é capaz de manter a taxa de desemprego abaixo da taxa natural, e o aumento dos preços resultante do processo não é eliminado. Em outras palavras, nesta concepção, há um viés inflacionário na condução da política monetária na tentativa de aumentar o nível de emprego.

No que se refere ao argumento empírico, as principais relações, que envolvem a independência do BC e a performance da economia, podem ser sumariadas da seguinte forma:

em economias industriais, independência legal e inflação apresentam forte correlação negativa

- em países com menor nível de desenvolvimento não há relação evi-

\footnotetext{
${ }^{4}$ Os trabalhadores decidem ofertar mais mão-de-obra porque esperam receber um salário real mais elevado que o de equilibrio correspondente à taxa natural de desemprego.

${ }^{5}$ Apesar deste ser um resultado comum para a literatura, Cargill (1995, p. 169, grifo meu), destaca que

The claimed statistical association between formal measures of central bank independence and inflation is contradicted by the inflation records of two of the most important central banks in
} 
dente entre independência legal e inflação;

- a taxa de inflação e a variação da mesma são positivamente relacionadas à vulnerabilidade política do BC;

- países, onde as autoridades monetárias têm anunciado suas metas, têm apresentado menores taxas de inflação;

- a independência legal dos bancos centrais não apresenta correlação com a média de crescimento real;

- bancos centrais com maior grau de autonomia não financiam déficits.

Os pontos acima destacados implicam que a inflação é mais baixa quanto mais independente for $\mathrm{o} \mathrm{BC}^{6}$, e que, dada a independência, países que preanunciam política monetária têm obtido menores taxas de inflação. E, ain$\mathrm{da}$, que flutuações da taxa de crescimento da economia parecem não estar relacionadas a $\mathrm{BCls}{ }^{7}$.

Um dos principais problemas na análise da proposição de independência dos bancos centrais refere-se à dificuldade em se avaliar quais os possiveis efeitos sobre a economia decorrentes de uma maior autonomia do $\mathrm{BC}$. $\mathrm{Na}$ busca de solução para este problema, diversos teóricos desenvolveram índices de independência para o $\mathrm{BC}^{8}$.

Em geral, a independência dos bancos centrais é medida por dois tipos de índices:

- os índices de independência legal - que procuram refletir a relação entre a política monetária e as leis que estabelecem e delimitam o poder dos bancos centrais; e

- os índices de independência com base em questionários (índices de independência real) - que se baseiam em julgamentos sobre o comportamento do $\mathrm{BC}$.

the world. The Bank of Japan, a formally dependent central bank, has generated a superior inflation record to the Federal Reserve, a formally independent central bank. Aside form this problem, the statistical association is not robust and subject to variation depending on countries included in the regression and regression specification. In summary [...] the claimed statistical association between low inflation and formal central bank independence should not be given the importance it has been afforded in discussions of how to structure a price stabilizing central bank.

${ }^{6}$ Diversos autores analisaram essa hipótese, entre eles destacam-se: Cukierman (1992); Cukierman, Webb e Neyapti (1992); Alesina e Summers (1993); Eijffinger e Schaling (1993), Eijfinger e von Keulen (1995).

${ }^{7}$ Ainda que relevante, a discussão sobre accountability e metas para a política monetária fogem ao escopo deste trabalho. Para uma discussão sobre esse tema, ver Haldane (1995); McDonough (1997); Mishkin e Posen (1997); e Haan, Amtenbrink e Eijffinger (1999).

${ }^{8}$ Para o leitor interessado na análise sobre índices de independência, recomenda-se como leitura: Bade e Parkin (1985); Alesina (1989); Cukierman (1992); Cukierman, Webb e Neyapti (1992); Alesina e Summers (1993); Eijffinger e Schaling (1993); Eijflinger e von Keulen (1995); Cukierman e Webb (1995); Cukierman (1996); e Forder (1999). 
É importante ressaltar que a literatura sobre o tema reconhece que, sem independência legal, o BC torna-se dependente das autoridades políticas. Assim, em um primeiro momento, é necessário a existência de independência legal para que seja criado um ambiente institucional adequado, capaz de criar condiçôes para que se observe maior independência por meio dos índices que se baseiam em questionários.

O objetivo do artigo consiste em apresentar as fragilidades associadas aos índices que medem o nível de independência do $\mathrm{BC}$. Os resultados encontrados denotam que maior grau de independência não é capaz de garantir baixas taxas de inflação. O trabalho apresenta-se estruturado da seguinte forma: a segunda seção mostra que o índice de independência de um determinado $\mathrm{BC}$ reflete a inflação da economia em que está inserido esse $\mathrm{BC}$, que a utilização do grau de independência não pode ser correlacionada com a inflação do mesmo período, e que há um caráter subjetivo à mensuração da independência por meio das divergências entre três índices existentes (Alesina-Summers, Cukierman-Webb-Neyapti e Grilli-Masciandaro-Tabellini). Na terceira seção, é feita uma analogia dos argumentos apresentados na seção anterior com os resultados oriundos da correlação entre os graus de independência do Banco Central do Brasil e as taxas de inflação nos anos 1980 e 1990; por último, a quarta seção contém as conclusões do artigo.

\section{A evolução dos índices de independência e suas fragilidades}

A análise empírica sobre a proposição de independência recebeu grande impulso entre a segunda metade da década de 80 e início dos anos 90 a partir do desenvolvimento de índices fundamentados em atributos legais que regem o $\mathrm{BC}^{9}$. Os primeiros autores a desenvolverem um índice para a independência do BC foram Bade e Parkin (1985) e Alesina (1989). As principais informações contidas nesses índices procuraram identificar três pontos básicos: a autoridade que controla a política monetária; a existência de funcionários do governo no board do $\mathrm{BC}$; e se a maior parte dos membros da diretoria do BC é nomeada pelo governo.

Nos anos 90, Grilli, Masciandaro e Tabellini (1991) elaboraram um

\footnotetext{
${ }^{9}$ Estes índices eram aplicados quase que de forma exclusiva para as economias industrializadas
} 
índice que abrange a maioria dos países industrializados, concentrandose em duas características:

- a independência de políticas - procedimento para nomeação dos membros da diretoria do banco, duração do mandato do Conselho, e a presença de diretrizes que imponha a busca da estabilidade monetária; e

- a independência econômica - o quanto a lei permite ao BC emprestar ao governo, e se a supervisão do sistema bancário encontra-se sob responsabilidade do $\mathrm{BC}^{10}$.

Seguindo o mesmo modelo desenvolvido por seus predecessores, Eijffinger e Schaling (1993) inseriram, na elaboração do índice, questões como a responsabilidade final pela política monetária, a presença de funcionários do governo na diretoria e a proporção de diretores nomeados pelo governo. A principal diferença em relação aos índices anteriores advém da relevância atribuída à responsabilidade da política monetária, sobretudo às leis que determinam o controle da política monetária como exclusiva do $\mathrm{BC}$.

Além dos índices supracitados, Cukierman, Webb e Neyapti (1992) elaboraram um índice de independência legal, referente ao período 195089 para todos os países industrializados e para cinqüenta economias em desenvolvimento.

Esse índice é construído a partir de 16 características legais básicas de cartaspatentes de bancos centrais, divididas nos seguintes quatro grupos:

a) a nomeação, demissão e duração legal do mandato do principal funcionário executivo do banco (normalmente o presidente);

b) a localização institucional da responsabilidade final pela política monetária e os procedimentos para a resolução de conflitos entre o governo e o $\mathrm{BC}$;

c) a importância da estabilidade de preços em comparação com outros objetivos, como nível elevado de emprego e estabilidade financeira;

d) o rigor e a universalidade das limitaçōes da capacidade do governo de tomar empréstimos do $\mathrm{BC}$, a taxas de mercado ou subsidiadas, ou de instruir o BC a emprestar a terceiros. (Cukierman, 1996, p. 395).

Embora exista uma diversidade razoável de índices para a mensuração do grau de independência, é reconhecido que "os índices existentes são indicadores de independência incompletos e cheios de ruído [...] o seu uso deverá ser suplementado pela crítica no contexto do problema em consideração" (Cukierman, 1996, p. 394). Destarte, deve-

${ }^{10}$ A questão do maior ou menor nível de supervisão do sistema bancário pelo banco central está associada à idéia do nível de esforço que o banco central implementa para a manutenção da estabilidade de preços 
se chamar atenção para o fato de que a tentativa de desenvolver a mensuração do grau de independência com base em índices já existentes não é um mecanismo confiável. Outrossim, observa-se que

[The] ... measures of independence are only acceptable if one already agrees with theoretical principles on which the thesis of correlation between independence and price stability is based. Of course, if one defends the ssignement of highest priority to price stability as a measure of independence and the correlates this measure of independence with price stability, one should not be surprised to find some correlation. (Carvalho, 1995/96, p. 169-170, grifo meu).

Tendo o propósito de evidenciar a tendência inerente aos questionários formulados para mensurar o grau de independência dos bancos centrais em refletir alta correlação negativa entre a independência do BC e a taxa de inflação, procura-se por meio da tabela a seguir, reunir as questões mais utilizadas para a mensuração dos índices de independência. O principal objetivo consiste em identificar alguma característica comum, a todas as perguntas, que seja capaz de revelar a preocupação apriorística na formulação dos índices existentes em obter a correlação supracitada.

Tabela 1: Perguntas mais freqüentes para mensuração da independência do Banco Central

\begin{tabular}{l|c|c|c|c|c}
\hline & BP & A & GMT & ES & CWN \\
\hline $\begin{array}{l}\text { I. O BC é o responsável final pela } \\
\text { política monetária? }\end{array}$ & $X$ & $X$ & $X$ & $X$ & $X$ \\
$\begin{array}{l}\text { 2. É obrigatória a presença de algum } \\
\text { membro do governo no board do BC? }\end{array}$ & $X$ & $X$ & $X$ & $X$ & $X$ \\
\hline $\begin{array}{l}\text { 3. Os membros do board do BC são } \\
\text { nomeados pelo governo? }\end{array}$ & $X$ & $X$ & $X$ & $X$ & $X$ \\
\hline $\begin{array}{l}\text { 4. Existem limitações quanto à } \\
\text { capacidade do governo de tomar } \\
\text { empréstimos do BC? }\end{array}$ & & $X$ & $X$ & & $X$ \\
\hline $\begin{array}{l}\text { 5. Existem cláusulas legais que fortalecem } \\
\text { o BC no caso de conflito com o governo? }\end{array}$ & & & $X$ & & $X$ \\
\hline $\begin{array}{l}\text { 6. Qual a importância da estabilidade de } \\
\text { preços em relação a outros objetivos? }\end{array}$ & & & $X$ & & $X$ \\
\hline $\begin{array}{l}\text { 7. Qual a duracão legal do mandato do } \\
\text { presidente do BC? }\end{array}$ & & & $X$ & & $X$ \\
\hline
\end{tabular}

$\mathrm{BP}=$ Bade e Parkin (1985);

$\mathrm{A}=$ Alesina (1989); $\quad$ GMT $=$ Grilli, Masciandaro $\mathrm{e}$ Tabellini (1991); $\quad$ ES = Eijffinger e Schaling (1993); CWN = Cukierman, Webb e Neyapti (1992). 
As questões contidas na tabela 1 podem ser divididas em três grupos:

i) perguntas $1,2,3$ (presentes em todos os índices em consideração) e 7 - têm por objetivo identificar qual a possivel influência do governo sobre as decisōes operacionais do $\mathrm{BC}$;

ii) perguntas 4 e 5 - têm o intuito de revelar a capacidade do $\mathrm{BC}$ em neutralizar as pressões oriundas do governo; $\mathrm{e}$

iii) pergunta 6 - tem a intenção de verificar se o BC está comprometido com a busca da estabilidade de preços ou com outros objetivos (por exemplo nível de emprego).

O ponto principal a ser destacado é que, apesar da divisão realizada, observa-se uma característica comum às perguntas. Todas foram construídas com o objetivo de avaliar qual o possivel viés inflacionário associado à condução da política monetária. Eis o principal elemento para uma crítica externa. Como as perguntas foram criadas, em última instância, para avaliar o impacto do maior grau de independência sobre a inflação (que se manifesta pela redução do viés inflacionário), é natural que os índices tenham a tendência de revelar correlação negativa entre maior independência do BC e inflação.

O corolário é que os índices existentes não servem como instrumento capaz de evidenciar os efeitos de maior independência do BC sobre a inflação. A justificativa advém do fato de que, se houver a preocupação do BC com a estabilidade de preços, isto implica baixo viés inflacionário, o que, por conseguinte, acarreta alto grau de independência. Por outro lado, se o objetivo do BC não for a estabilidade de preços, tende a ocorrer um aumento no viés inflacionário, o que leva à queda no grau de independência. Dessa forma, observa-se um caráter endógeno nos índices. Quando a economia apresenta baixo nível de inflação, há uma propensão dos índices revelarem maior nível de independência do $\mathrm{BC}$, ao passo que, quando a inflação é elevada, os mesmos índices apresentam baixo grau de independência. Logo, é o nível de inflação de uma economia que determina o grau de independência do $\mathrm{BC}$ e não o contrário.

A forma mais simples para observar a possivel influência de um BCI sobre a inflação é o uso de gráficos de dispersão. Na grande maioria das vezes, estes gráficos revelam de forma simplificada uma correlação negativa entre o grau de independência e a inflação. Apesar deste instrumento apresentar resultados que são confortáveis para os defensores de um $\mathrm{BCI}$, pode-se fazer uma crítica interna. Mesmo que se considere razoáveis os índices existentes, há um elemento que é negligencia- 
do pela maioria dos economistas que discutem o assunto - o fato de o grau de independência de um determinado período não poder ser utilizado sobre a média da inflação no mesmo período. Este argumento deriva da observação de que maior independência hoje não significa menores taxas de inflação no mesmo momento. Para que exista independência é preciso credibilidade ${ }^{11}$, o que implica a necessidade de incorporar a variável tempo na análise, ou seja, para que se possa avaliar os possíveis efeitos de maior independência do BC, é preciso verificar o comportamento da inflação após um determinado período.

Deve-se juntar aos pontos destacados acima o fato de que a simples existência de correlação pode não implicar causalidade.

Posen (1993) [...] demonstrou que a relação normalmente encontrada entre grau de independência do $\mathrm{BC}$ e taxas de inflação não é causal. Após a realização de testes econométricos afirmou que países onde o conjunto de interesses é de cunho antiinflacionário constroem instituições para sustentar sua aversão e países onde predominam interesses coniventes com a inflação não desenvolvem tais instituições. Aduziu, ainda, que existe uma clara relação causal entre interesses antiinflacionários mais efetivos e maior independência do $\mathrm{BC}$, e nāo entre independência do $\mathrm{BC}$ e baixa inflação. (Sicsú, 1996, p. 26).

Ademais, um outro aspecto, a ser considerado, consiste em saber se os índices tratam do mesmo objeto. Este é um ponto importante, pois os índices foram criados para mensurar a independência do BC. Assim, se a concepção do que é independência difere de índice para indice, isto significa que não há um conceito homogêneo para independência. Assumindo-se que os índices são derivados de conceitos distintos, os resultados obtidos da análise entre grau de independência e diversos elementos na economia (taxa de juros, produto, inflação, etc.) podem não ser unívocos e, portanto, gerar resultados inconclusivos para a análise empírica. Esta observação é apontada como uma das principais fragilidades para a análise empírica sobre independência.

\footnotetext{
${ }^{11} \mathrm{O}$ conceito credibilidade pode ser entendido como o nível de confiança que os agentes econômicos depositam na exequibilidade de uma política anunciada ser implementada e ser cumprida até o fim. Ou seja, uma politica inspirará maior credibilidade se ela sinalizar aos agentes uma chance reduzida da ocorrência de inconsistência temporal. Assim, se, por exemplo, o BC ao longo de sua história obteve êxito no combate à inflação (o que implica conquista de reputação) os agentes acreditam que o $\mathrm{BC}$ terá sucesso no controle da inflação futura, o que, por sua vez, denota alto grau de credibilidade.
} 
The failure to agree on a measure of independence makes impossible ary test of the effects of independence. It is important recognize exactly what the point is. It is not the foolish one that different authors use different approaches to measure independence and so cannot be said to be talking about the same thing. The problem is not the different approaches to measurement, but rather the different resultant measures.

[...] The claim of these studies [measurement of independence] is that they show a general tendency for lower inflation to be associated with greater independence. And indeed this claim could even be correct, but what the literature has not succeeded in doing is offering an empirical concept of independence that allows the claim to be tested. Forder (1999, p. 35, 36, grifo meu).

Uma forma simples de testar se os índices referem-se ao mesmo objeto consiste na análise dos coeficientes de correlação entre diversas medidas de independência. Para tanto, optou-se pela seleção de três índices que são utilizados com grande freqüência nos estudos de natureza empírica sobre independência do BC: Alesina-Summers (AS), Cukierman-Webb-Neyapti (CWN) e Grilli-Masciandaro-Tabellini (GMT).

Tabela 2:

\begin{tabular}{lcccccc}
\hline \multicolumn{1}{c}{ Países } & AS & Rank & CWN & Rank & GMT & Rank \\
\hline Alemanha & 4 & 1 & 0,69 & 1 & 13 & 1 \\
Austrália & 2 & 4 & 0,36 & 6 & 9 & 4 \\
Bélgica & 2 & 4 & 0,17 & 13 & 7 & 6 \\
Canadá & 2,5 & 3 & 0,45 & 5 & 11 & 3 \\
Dinamarca & 2,5 & 3 & 0,5 & 3 & 8 & 5 \\
Espanha & 1,5 & 6 & 0,23 & 11 & 5 & 8 \\
França & 2 & 4 & 0,24 & 10 & 7 & 6 \\
Itália & 1,75 & 5 & 0,25 & 9 & 5 & 8 \\
Japão & 2,5 & 3 & 0,18 & 12 & 6 & 7 \\
Noruega & 2 & 4 & 0,17 & 13 & $n / a$ & $n / a$ \\
Nova Zelândia & 1 & 7 & 0,24 & 10 & 3 & 9 \\
Reino Unido & 2 & 4 & 0,27 & 8 & 6 & 7 \\
Suécia & 2 & 4 & 0,29 & 7 & $n / a$ & $n / a$ \\
EUA & 3,5 & 2 & 0,48 & 4 & 12 & 2 \\
Suíça & 4 & 1 & 0,64 & 2 & 12 & 2 \\
\hline
\end{tabular}

Os dados contidos na tabela 2 permitem a elaboração dos gráficos (1.A), (1.B) e (1.C). Tais gráficos evidenciam a existência de forte correlação positiva entre as três medidas de independência. Este tipo de resultado sinaliza que os índices sob análise convergem quanto à mensuração do nível de independência dos bancos centrais, o que, por conseguinte, leva à conclusão aparente de que não há divergências quanto aos resultados das diversas metodologias empregadas para a elaboração dos índices de independência. 


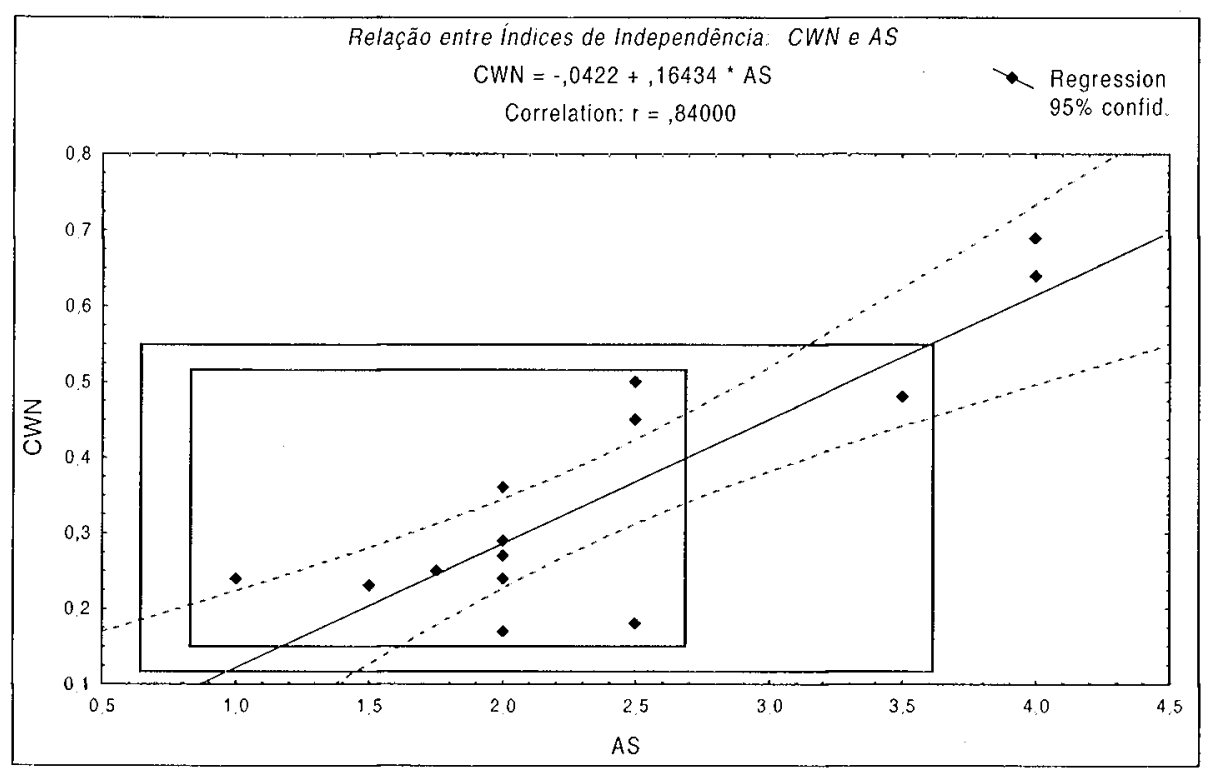

Gráfico 1.A

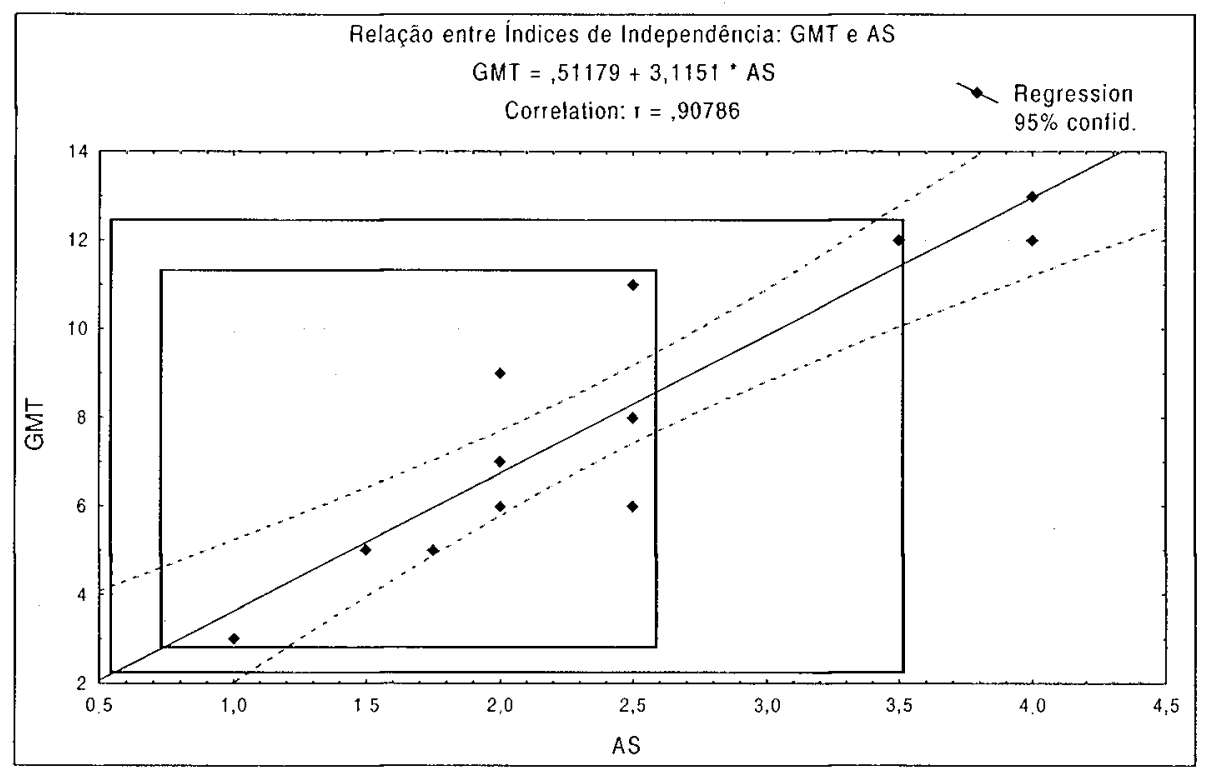

\section{Gráfico 1.B}




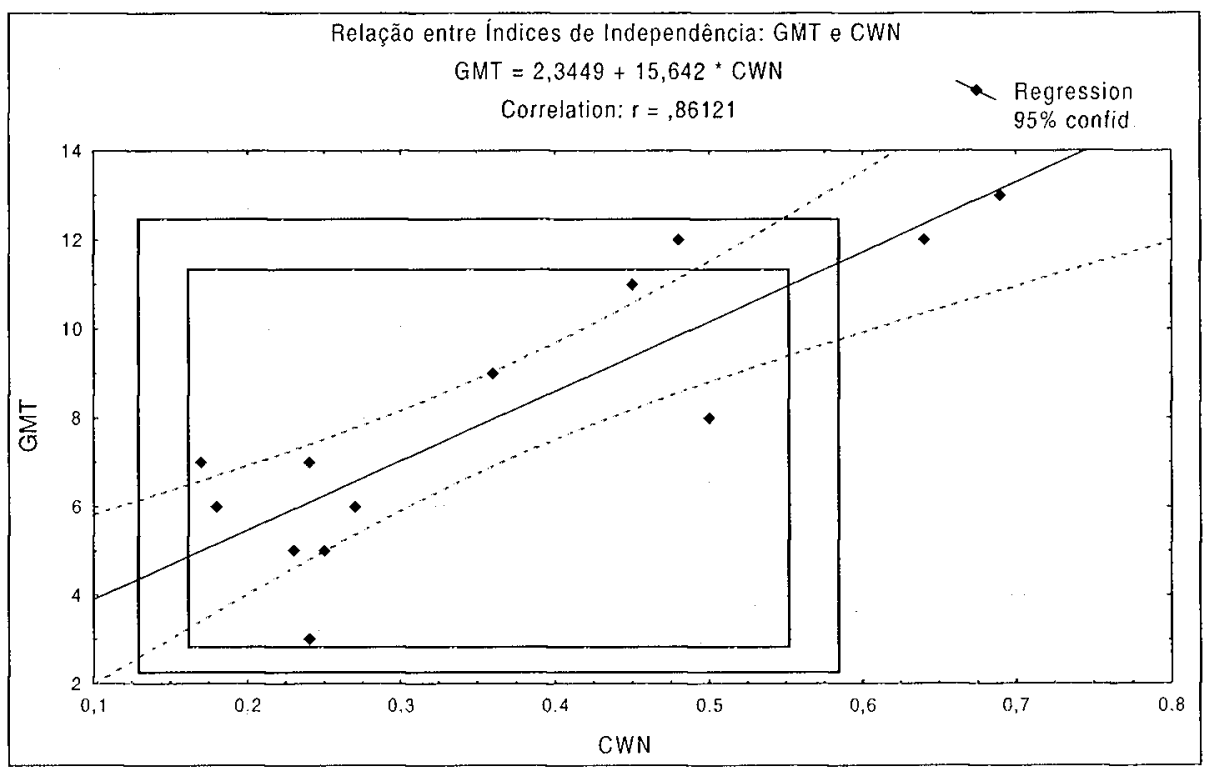

Gráfico 1.C

Apesar de o resultado obtido (convergência na mensuração da independência) mostrar-se satisfatório do ponto de vista estatístico, observa-se que o rank de independência do BC associado a cada metodologia apresenta dissensões que não podem ser desprezadas (ver tabela 2). É importante lembrar que os testes de correlação entre os índices não são capazes de identificar esse problema, uma vez que eles apenas relacionam os pesos dos coeficientes sem qualificar a que pontos se referem. Em outras palavras, os testes de correlação apenas indicam que dentro da amostra selecionada há uma distribuição de pontos que é semelhante, mas isto não significa que a classificação do nível de independência de cada país seja similar entre os índices selecionados. Destarte, uma forma alternativa de verificar a existência de coesão entre os índices, tendo a preocupação qualitativa, consiste na realização dos testes de correlação entre os ranks associados a cada índice.

Os gráficos (2.A), (2.B) e (2.C) ilustram a correlação entre os diversos ranks. Da mesma forma que no caso anterior, as correlações encontradas para os três casos são significativas. Entretanto, mais uma vez, os resultados encontrados merecem atenção. Conforme destacado por Forder (1999, p. 33) "Clearly, the apparent similarity of measures of independence depends on agreement that the central banks of Germany and Switzerland are highly independent". 


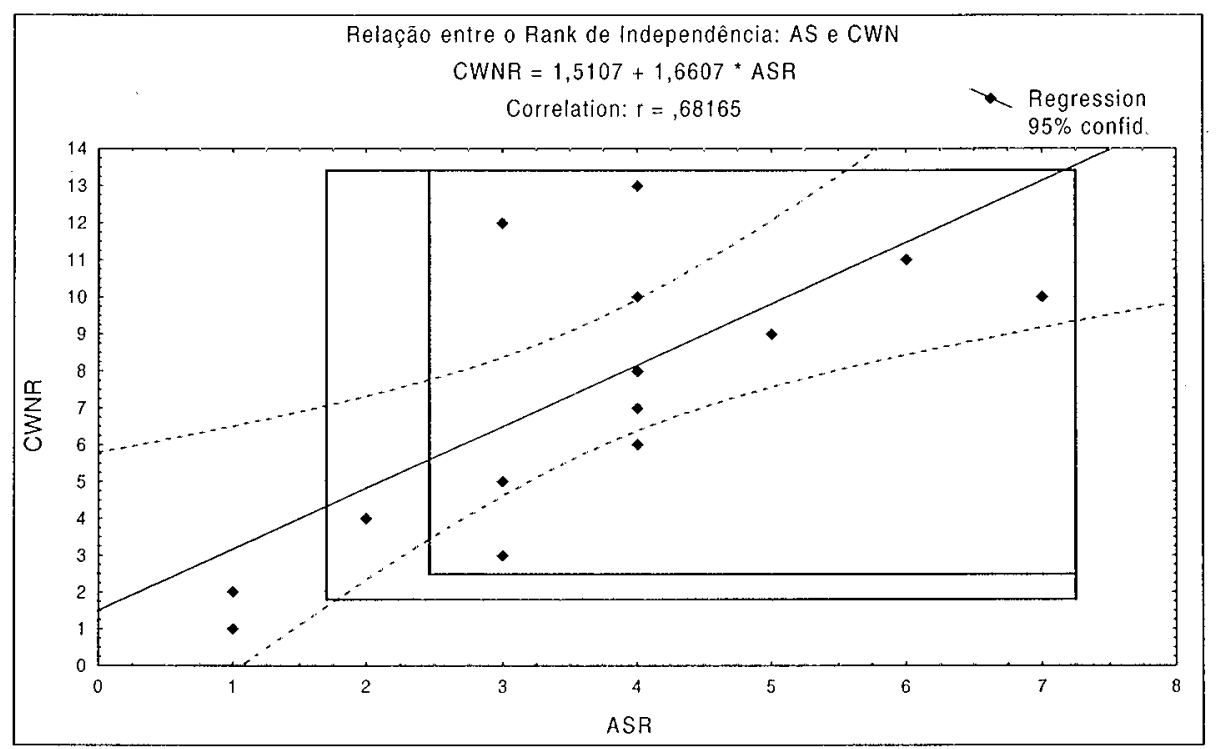

\section{Gráfico 2.A}

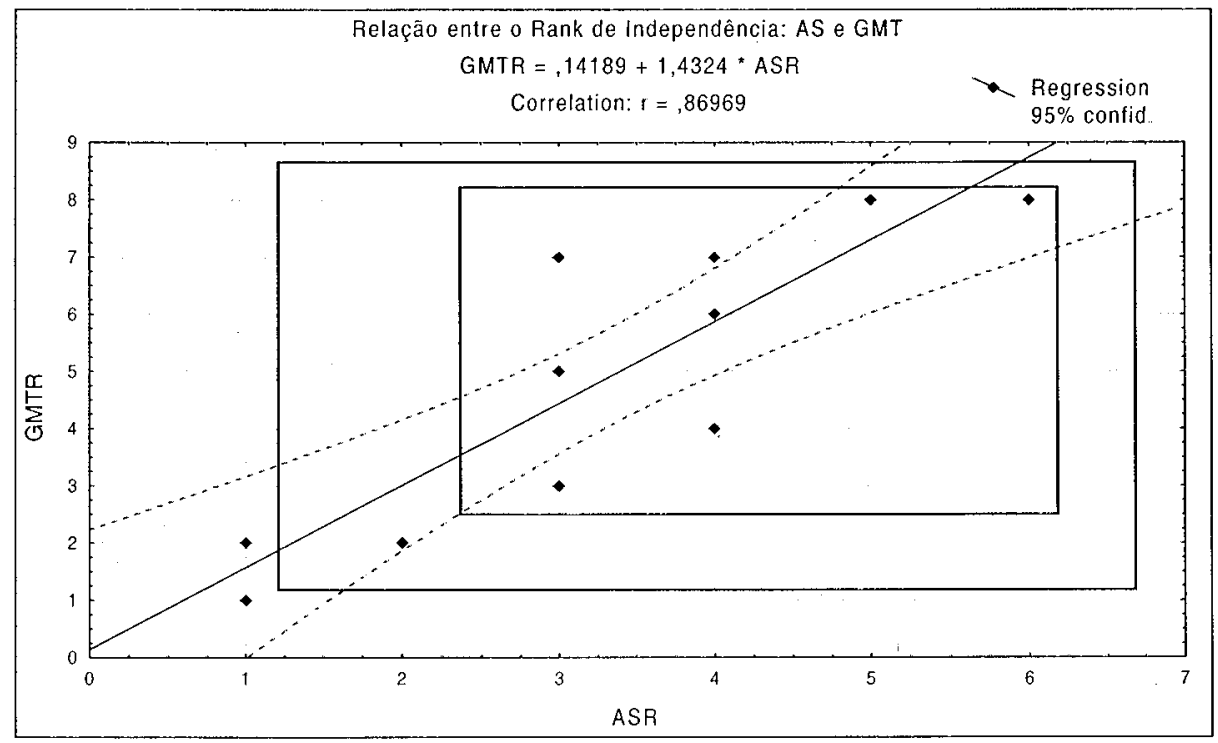

\section{Gráfico 2.B}




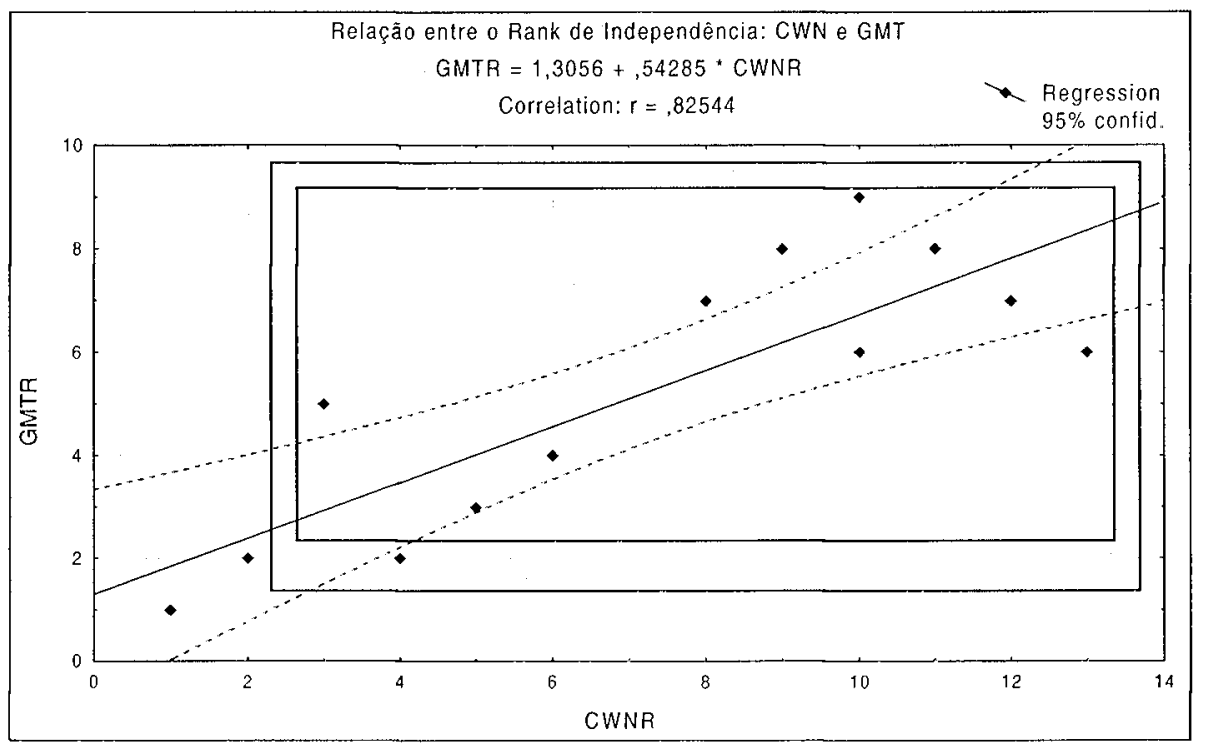

\section{Gráfico 2.C}

É importante observar que, se forem excluídos da análise os países que representam consenso para os três índices no que se refere ao rank de independência dos bancos centrais (Alemanha e Suíça), ilustrado pela área contida no retângulo maior dos gráficos (1.A), (1.B), (1.C), (2.A), (2.B) e (2.C), os resultados obtidos não são unívocos ${ }^{12}$. Os coeficientes de determinação $\left(R^{2}\right)$ encontrados para a relação entre os índices e o rank Alesina-Summers e Cukierman-Webb-Neyapti tornam-se pouco significativos (ver tabela 3).

Além do Bundesbank e do Banco Nacional da Suíça, outro BC que é normalmente destacado pela literatura como paradigma de independência é o Federal Reserve System (FED). Este dado merece consideração, pois, se forem retirados da amostra, Alemanha, Suíça e EUA (nos gráficos supracitados corresponde à área do retângulo menor), os coeficientes de determinação observados para a relação entre os três índices e ranks de independência sofrem queda significativa (ver tabela 3). Assim, as evidências obtidas sugerem que, quando são eliminados da amostra os países que apresentam um histórico de sucesso no combate à inflação, não se obtém uma ordenação razoável para a independência dos diversos bancos centrais. Por conseguinte, uma análise que en-

${ }^{12}$ A estratégia de análise utilizada baseia-se no argumento que foi destacado na nota de rodapé 5. 
volva um conjunto de países que exclua Alemanha, Suíça e EUA pode levar a resultados inconclusivos.

Tabela 3:

\begin{tabular}{|c|c|c|c|c|c|}
\hline Índices & Correlação & $\mathrm{R}_{2}$ & Rank & Correlação & $\mathrm{R}_{2}$ \\
\hline (AS-CWN) & 0,8400 & 0,7056 & $A S(R)-C W N(R)$ & 0,6816 & 0,4646 \\
\hline$\left(\mathrm{AS}-\mathrm{CWN|^{* }}\right.$ & 0,6059 & 0,3671 & {$[A S[R]-C W N[R]]^{*}$} & 0,4599 & 0,2115 \\
\hline$\left(A S-\left.C W N\right|^{* *}\right.$ & 0,4290 & 0,1841 & {$\left[A S[R]-C W N(R]^{* *}\right.$} & 0,3327 & 0,1107 \\
\hline (AS - GMT) & 0,9079 & 0,8242 & $A S(R)-G M T(R)$ & 0,8898 & 0,7917 \\
\hline$(A S-G M T)^{*}$ & 0,8547 & 0,7305 & {$[A S(R] \cdot G M T(R)]^{*}$} & 0,8159 & 0,6657 \\
\hline$(A S \cdot G M T)^{* *}$ & 0,7626 & 0,5816 & {$[A S[R]-G M T[R]]^{* *}$} & 0,7522 & 0,5658 \\
\hline$(C W N-G M T)$ & 0,8612 & 0,7417 & $C W N(R)-G M T(R)$ & 0,8254 & 0,6813 \\
\hline$(C W N \cdot G M T)^{*}$ & 0,7566 & 0,5725 & {$[C W N(R)-G M T(R)]^{*}$} & 0,7057 & 0,4980 \\
\hline$(C W N-G M T)^{* *}$ & 0,6685 & 0,4469 & {$[C W N[R]-G M T(R)]^{* *}$} & 0,6235 & 0,3887 \\
\hline
\end{tabular}

(*) exclui Alemanha e Suíça

$\left({ }^{* *}\right)$ exclui Alemanha, Suíça e EUA.

Além do que foi destacado, verifica-se que a divergência entre os índices revela um caráter subjetivo à mensuração do grau de independência, por conseguinte, as implicações derivadas a partir dos mesmos não podem ser consideradas robustas. A justificativa é simples, o grau de independência é uma estimativa, portanto, é intrínseco ao grau de independência a possibilidade da existência de erro, o que, por sua vez, pode comprometer os resultados que se baseiam nos índices de independência. Deve-se ressaltar que, mesmo que se admita como razoáveis as estimativas dos índices, observa-se que os resultados não são homogêneos (ver análise para os ranks de independência), o que de forma indubitável coloca em xeque os resultados empíricos que se sustentam em tais estimativas.

\section{Evidências empíricas do Banco Central do Brasil: um caso ilustrativo}

$\mathrm{Na}$ ânsia de encontrar resultados robustos para justificar que maior independência do BC implica menor nível de inflação, a quase totalidade dos estudos realizados utilizou graus de independência e taxas de inflação de diversos países na mesma análise. Este artifício técnico possibilita a manipulação de dados para a confirmação da idéia de que, "legal independence is systematically and inversely related to inflation in industrial, but not in developing, countries" (Cukierman, Webb e Neyapti, 1992, p. 376, grifo meu). Além disso, a utilização da técnica citada não permite observar qual o efeito sobre o nível de preços resul- 
tante do esforço em aumentar a independência do BC de um determinado país.

Como forma de se observar se o aumento da independência do BC é capaz de estabilizar preços para uma economia em particular, fazse a seguir um exame do caso brasileiro. O Brasil foi selecionado para análise porque desde o final dos anos 80 tem sido observado um aumento no grau de independência do Banco Central do Brasil (BCB). Ademais, de acordo com Cukierman, Webb 83 Neyapti (1992), o país se enquadra no caso de países em desenvolvimento, representando um elemento do conjunto de países em que não há evidências de que independência legal afete o nível de inflação.

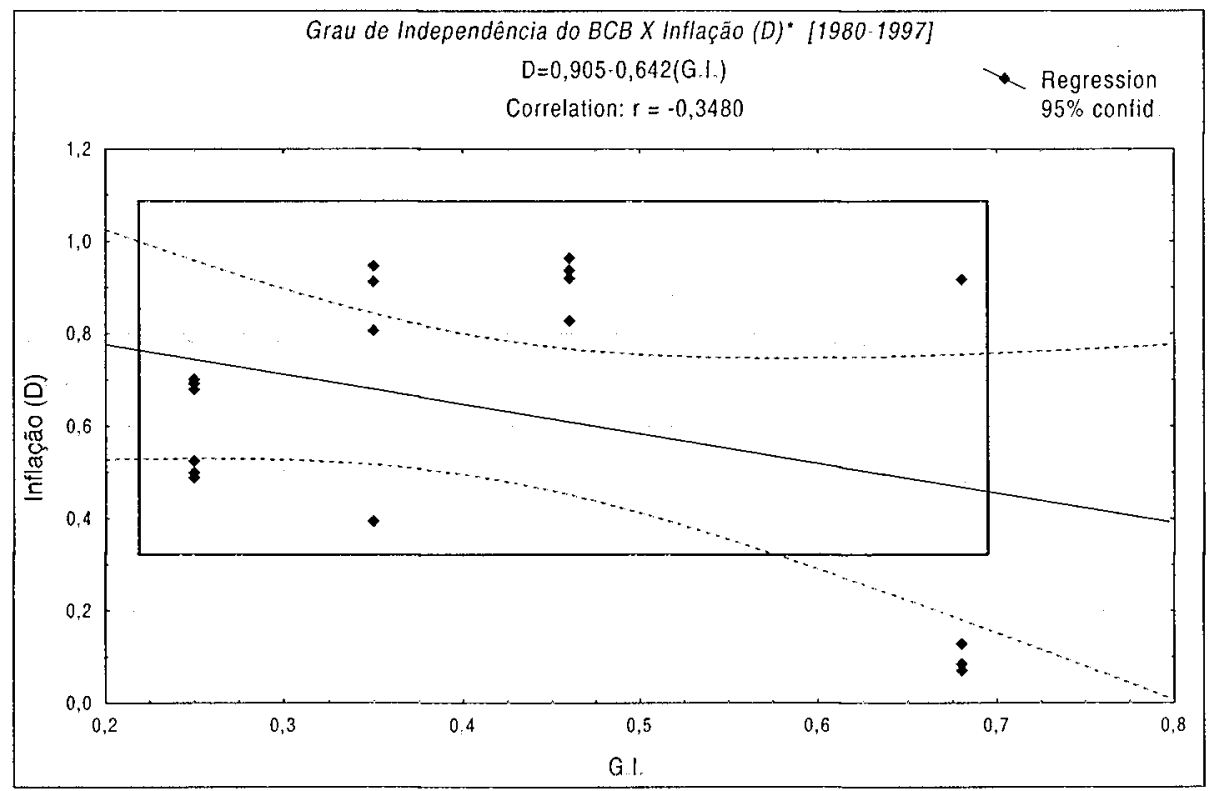

\section{Gráfico 3}

Obs: $0 £$ G.I £ 1. A inflaçāo corresponde à média anual do IGP-DI sendo transformada pela fórmula $D=p /(1+p)$ para reduzir a heterocedasticidade do erro e melhorar a eficiência do estimador.

Fazendo-se uso do grau de independência do BCB obtido por meio de questionário ${ }^{13}$ e a média da inflação (ver gráfico 3), observa-se

${ }^{13}$ A mensuração do grau de independência do $\mathrm{BCB}$ teve por base um questionário [adaptaçăo do modelo elaborado por Cukierman (1992), ver também Cukierman, Webb e Neyapti (1992)] submetido a todos os presidentes do Banco Central do Brasil [quanto à metodologia empregada, ver Rigolon (1997)]. 
que os argumentos destacados anteriormente são corroborados. A correlação negativa entre o grau de independência e a inflação para o caso brasileiro evidencia o viés existente nos îndices que medem o nível de independência do $\mathrm{BC}$. A justificativa provém do fato de que o sucesso do Plano Real no combate à inflação foi o responsável pelo aumento do grau de independência. Em termos do gráfico em consideração, este resultado pode ser observado pela eliminação da correlação negativa quando se retira da amostra o período posterior a 1994 (área contida no retângulo do gráfico 3$)^{14}$.

Dado que a existência de correlação não necessariamente implica causalidade, o gráfico acima pode indicar uma leitura do tipo: maior independência do BCB indica menores taxas de inflação; ou menores taxas de inflação indicam maior grau de independência. Para que não haja dúvidas quanto à afirmação correta, basta observar a equação de regressão e os pontos no gráfico 3. Não obstante a existência de correlação negativa entre grau de independência e inflação, a maioria dos pontos encontra-se fora da região que corresponde à hipótese de que maior independência do BCB implica menor nível de inflação.

O gráfico 4 mostra o comportamento da inflação ${ }^{15} \mathrm{e}$ do grau de independência do BCB para o período 1986-96. Observa-se que houve uma elevação gradativa do grau de independência no período. Entretanto, esse aumento não foi suficiente para conter a trajetória ascendente da inflação. Nesse sentido, o trade-off grau de independência e nível de inflação para o período pré-1994 não existiu, o que indica não ter havido uma possivel relação causal entre maior grau de independência e inflação para esse período.

Deve-se destacar que o grau de independência do BCB para o período 1994/96 foi significativo (0,68 para um máximo de 1). Entretanto, verifica-se que os elementos-chave para esse resultado são fruto da própria decisão do governo (preocupação com a estabilidade de preços, a manutenção da âncora cambial, o processo de privatizações, redução dos gastos governamentais, etc.), ou seja, o governo foi responsável pela criação

\footnotetext{
${ }^{14} \mathrm{O}$ leitor mais atento deve ter percebido que este resultado está completamente de acordo com a crítica externa apresentada na seção anterior. Além disso, observa-se que o argumento presente em Cukierman, Webb e Neyapti (1992) e Cukierman e Webb (1995) de que países em desenvolvimento, inclusive o Brasil, deveriam substituir o índice de independência legal por um índice de rotatividade, para que fosse possível observar alguma relação entre independência e inflação, não se confirma

${ }^{15}$ Variaçāo mensal do Índice Geral de Preços (Disponibilidade Interna) da Fundação Getúlio Vargas.
} 
de um cenário econômico em que o BCB coopera na obtenção das metas pretendidas pelo governo. Como é fácil de observar, isso não significa maior independência do $\mathrm{BC}$, pois houve uma clara subordinação do $\mathrm{BCB}$ ao governo como forma de dar sustentação ao Plano Real. O que mais uma vez ratifica o caráter endógeno do grau de independência do $\mathrm{BC}^{16}$.

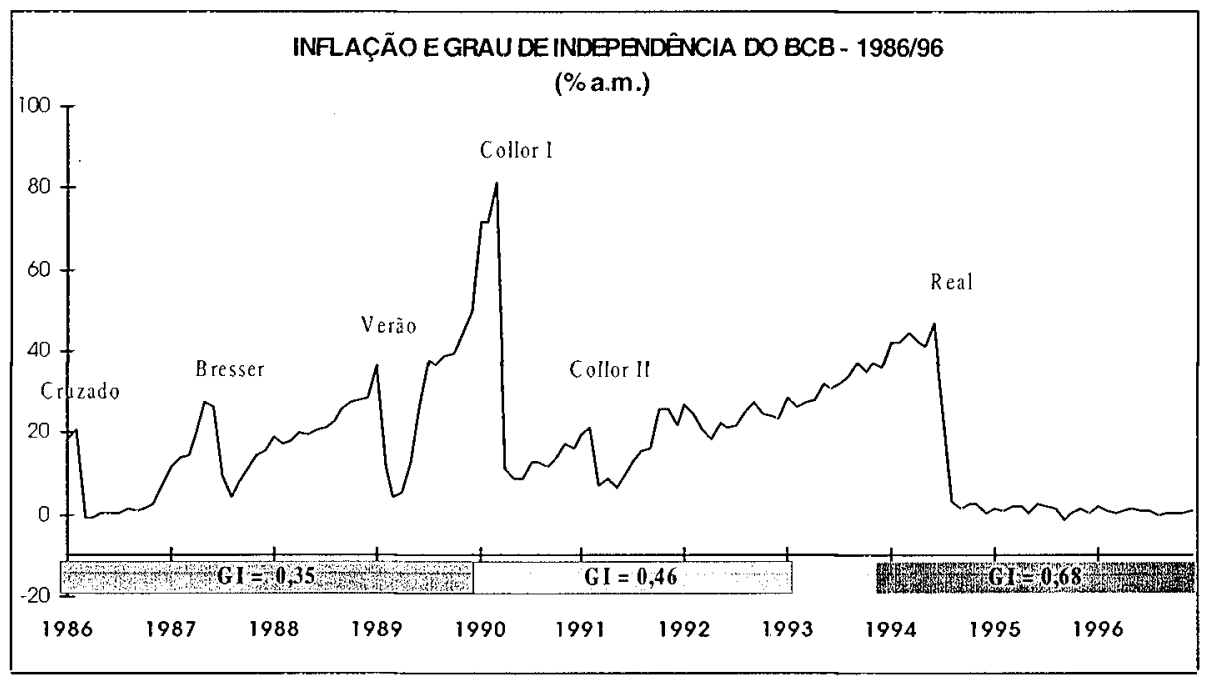

\section{Gráfico 4}

Fontes: Banco Central do Brasil e Rigolon (1997) ${ }^{17}$.

\section{Conclusões}

A análise realizada permite concluir que os índices existentes são viesados, não constituindo um instrumento capaz de revelar de forma razoável a relação independência-inflação. Também foi observado que a forma como os índices são utilizados para a análise entre independência e inflação é inadequada, pois ignora o tempo de resposta da ação da política monetária sobre a economia. Ademais, verificou-se

\footnotetext{
${ }^{16}$ Este resultado é compativel com o argumento destacado por Posen (1995) de que instituiçōes como o banco central são endógenas.

${ }^{17}$ Deve-se destacar que o grau de independência para o ano de 1993 não está disponível. Como o autor deste trabalho decidiu utilizar os graus de independência disponiveis em Rigolon (1997), achou-se conveniente não efetuar o cálculo para o referido ano para que não ocorressem divergências quanto à metodologia utilizada.
} 
que os índices expressam diferentes conceitos de independência. O que existe de fato é um consenso no que se refere às características dos países, cujo desenho de um BC estão associados à baixa taxa de inflação.

Não basta a literatura que discute o tema mostrar que Bundesbank, Banco Nacional da Súça e FED representam modelos de independência do $\mathrm{BC}$, e que, devido a essa estrutura, recebem a dádiva da credibilidade capaz de garantir a estabilidade de preços. O que dizer de países como o Japão, com um BC dependente e com taxas de inflação inferiores a dos EUA? Ou ainda dos casos de Portugal e Grécia, classificados como países com razoável grau de independência $(0,41 \mathrm{e}$ 0,55 respectivamente - CWN), mas que apresentam taxas de inflação consideradas elevadas para o padrão europeu. Conforme lembrado recentemente por Forder (1999, p. 38, grifo meu) "Eliminating the outliers without a clearly convincing reason is not science".

\section{Referências bibliográficas}

AKHTAR, M. A. "Monetary Policy Goals and Central Bank Independence”. BNL Quaterly Review, n 195, December 1995.

ALESINA, A. e SUMMERS, L. "Central Bank Independence and Macroeconomic Performance: Some Comparative Evidence". Journal of Money, Credit and Banking, May 1993.

BADE, R. e PARKIN, M. Central Bank Lows and Monetary Policy. Department of Economics, University of Western Ontario, 1985.

BLINDER, A. "What Central Bankers Could Learn from Academics - and Vice Versa”. Journal of Economic Perspectives. V. 11, n 2, Spring 1997.

CARGILL, T. F. "The Statistical Association between Central Bank Independence and Inflation". Banca Nazionale del Lavoro Quaterly Review, n. 193, June 1995.

CARVALHO, F “The Independence of Central Banks: a Critical Assesment of the Arguments". Journal of Post Keynesian Economics / Winter 1995-96, vol. $18, \mathrm{n}^{\circ} 2$.

CUKIERMAN, A. "A Economia do Banco Central". Revista Brasileira de Economia, Rio de Janeiro, 50(4), out./dez. 1996.

"Central Bank Independence and Monetary Control". The Economic Journal, 104, November 1994. 
Central Bank Strategy, Credibility, and Independence: Theory and Euidence. Cambridge, Mass.: The MIT Press, 1992.

CUKIERMAN, A., WEBB, S., e NEYAPTI, B. "Measuring the Independence of Central Banks and Its Effects on Policy Outcomes". World Bank Economic Review, 6(3), 1992.

CURRIE, D., LEVINE, P., e PEARLMAN, J. "The Choice of 'Conservative' Bankers in Open Economies: Monetary Regime Options For Europe". The Economic Journal, March, 1996, p. 345-358.

EIJFFINGER, S. e HAAN, J. The Political Economy of Central Bank Independence. Princeton University: Special Papers in International Economics, $\mathrm{n}^{\circ}$ 19, May 1996.

EIJFFINGER, S., e SCHALLING, E. "Central Bank Independence in Twelve Industrial Coutries”. Banca Nazzionale del Lavoro Quaterly Review, March, 1993.

EIJFFINGER, S. e VAN KEULEN, M. “Central Bank Independence in Another Eleven Countries”. Banca Nazionale Del Lavoro Quaterly Review, $\mathrm{n}^{\circ}$ 192, March 1995.

FISCHER, S. "Central Bank Independence Revisited". The American Economic Review, May 1995.

FORDER, J. "Central Bank Independence: Reassessing the Measurements”. Journal of Economic Issues. Vol. XXXIII, n 1 , March 1999.

GRILLI, V., MASCIANDARO, D. E TABELLINI, G. "Political and Monetary Institutions and Public Financial Policies in the Industrial Countries". Economic Policy, 13, October 1991.

HAAN, J., AMTENBRINK, F. e EIJFFINGER, S. "Accountability of Central Banks: Aspects and Quantification”. BNL Quaterly Review, n²09, June 1999.

HAAN, J., KNOT, K. e STURM, E. "On the Reduction of Disinflation Costs: Fixed Exchange Rates or Central Bank Independence?" BNL Quaterly Review, n 187, December 1993.

HAHN, J. e STURM, J. "The Case of Central Bank Independence". Banca Nazionale del Lavoro Quaterly Review, September 1992.

HALDANE, A. Targeting Inflation. Bank of England, 1995.

JENKINS, M.A. “Central Bank Independence and Inflation Performance: Panacea or Placebo?” BNL Quaterly Review, n 197, June 1996. 
JENSEN, H. "Credibility of Optimal Monetary Delegation”. American Economic Review, December 1997.

McDONOUGH, W. "A Framework for the Pursuit of Price Stability". Economic Policy Review. Federal Reserve Bank of New York, V. 3, n³., August 1997.

MISHKIN, F. e POSEN, A. "A Framework for the Pursuit of Price Stability". Economic Policy Review. Federal Reserve Bank of New York, V. 3, nº 3., August 1997.

PERSON, T. e TABELLINI, G. "Designing Institutions for Monetary Stability". (In) Persson e Tabellini (ed.) Monetary and Fiscal Policy. MIT Press, 1994.

POSEN, A. "Declarations are Not Enough: Financial Sector Sources of Central Bank Independence". NBER Macroeconomics Annual, 1995.

- "Why Central Bank Independence Does Nor Cause Low Inflation: There Is No Institutional Fix for Poliics". The Amex Bank Review, p. 40-65, 1993.

RIGOLON, F.J.Z. "Independência do Banco Central: Teoria e Aplicações para o Brasil". Ensaios BNDES 3, abril 1997.

SICSÚ, J. Uma Crítica à Tese da Independência do Banco Central. Nova Economia, Belo Horizonte, V. 6, nº 2, nov. 1996. 